\title{
Indicadores de desempenho motor como preditores de fragilidade em idosos cadastrados em uma Unidade de Saúde da Família
}

\author{
Motor performance indicators as fragility predicts in elderly registered in a \\ Family Health Unit
}

Priscila Luzia de Souza Santos ${ }^{1}$, Marcos Henrique Fernandes ${ }^{1}$, Patrícia Honório Silva Santos ${ }^{1}$, Tamiles Daiane Borges Santana ${ }^{1}$, Cesar Agusto Cassoti ${ }^{1}$, Raildo da Silva Coqueiro ${ }^{1}$, José Ailton Oliveira Carneiro ${ }^{1^{*}}$

ARTIGO ORIGINAL | ORIGINAL ARTICLE

\begin{abstract}
Este estudo objetivou identificar os testes de desempenho motor mais fortemente associados à fragilidade e seus respectivos pontos de corte, e verificar o melhor indicador de desempenho motor como instrumento de triagem para discriminar fragilidade em idosos cadastrados em Unidade de Saúde da Família. Participaram da pesquisa 139 idosos com idade $\geq 60$ anos, sendo 105 mulheres e 34 homens. A fragilidade foi identificada por meio dos critérios de Fried et al. (2001). Os testes de desempenho motor realizados foram: Força de preensão manual (FPM), sentar e levantar, caminhada (TC) e pegar um lápis. A análise de regressão logística foi usada para associar os testes de desempenho motor e fragilidade. Os pontos de corte foram avaliados por parâmetros fornecidos pela curva Receiver Operating Characteristic (ROC), com nível de significância de 5\%. Os dados foram analisados usando SPSS 21.0 e MedCalc. A média de idade dos idosos foi de $72.32 \pm 8.4$ anos. O teste de caminhada foi positivamente associado à fragilidade (OR 1,30; $\mathrm{p}<0.01$ ), e a força de preensão manual foi inversamente associada à fragilidade no sexo feminino (OR 0.74; $\mathrm{p}<0.001$ ). O TC apresentou um ponto de corte 5 s (sensibilidade 88,9 e especificidade $74,5 \%$ ) e a FPM-F obteve um ponto de corte de $14.6 \mathrm{kgf}$ (sensibilidade 83.3 e especificidade $79.0 \%$ ). Conclui-se que o teste de caminhada foi o melhor indicador de triagem para discriminar a fragilidade em idosos, de ambos os sexos, cadastrados em uma Unidade de Saúde da Família.
\end{abstract}

Palavras-chave: Idosos, fragilidade, desempenho motor.

ABSTRACT

This study aimed to identify the motor performance tests more strongly associated with frailty and respective cut-off points, and verify the best motor performance indicator as screening tool to discriminate frailty in elderly registered in Family Health Unit. Participated in the study 139 elderly aged $\geq 60$ years, 105 women and 34 men. The frailty was identified by the criteria of Fried et al. (2001). The motor performance tests realized were: Handgrip strength (HS), sit-to-stand test, walk test (WT) and pick up a pen test. Logistic regression analysis was used to associate the motor performance tests and frailty. The cutoff points were evaluated by parameters provided by Receiver Operating Characteristic curve (ROC), with significance level of 5\%. Data were analyzed using SPSS 21.0 and MedCalc. The mean age was $72.32 \pm 8.4$. The walk test was positively associated with frailty (OR 1.30; $<<0.01$ ), and handgrip strength in elderly women was inversely associated with frailty on feminine sex (OR 0.74; $\mathrm{p}<0.001$ ). The WT presented a cutoff $5 \mathrm{~s}$ (sensitivity 88.9 and specificity $74.5 \%$ ) and the handgrip in the women obtained a cutoff point $14.6 \mathrm{kgf}$ (sensitivity 83.3 and specificity $79.0 \%$ ). It was concluded that the walk test was the best screening indicator to discriminate the frailty in elderly, both sexes, registered in a Family Health Unit.

Keywords: Elderly, frailty, motor performance.

Artigo recebido a 29.10.2015; Aceite a 17.05.2016

${ }^{1}$ Universidade Estadual do Sudoeste da Bahia - UESB.

* Autor correspondente: Universidade Estadual do Sudoeste da Bahia. Núcleo de Estudos em Epidemiologia do Envelhecimento. Rua José Moreira Sobrinho, s/nº - Jequiezinho. CEP 45206-190 - Jequié, BA, Brasil. Tel: (73) 3528-9600.. E-mail: hitoef@yahoo.com.br 


\section{INTRODUÇÃO}

Em paralelo ao processo de envelhecimento ocorrem inúmeras alterações que comprometem as condições de saúde dos idosos, aumentando a prevalência de doenças crônicas e síndromes geriátricas, dentre as quais pode-se observar a Síndrome da Fragilidade (Reis Júnior, Carneiro, Coqueiro, Santos, \& Fernandes, 2014).

A fragilidade é considerada uma síndrome clínica geriátrica de condição multissistêmica, sendo caracterizada pelo aumento da vulnerabilidade a estressores, diminuição das reservas fisiológicas e desequilíbrios de múltiplos sistemas (Fried et al., 2001; Fried et al., 2004, \& Walston, 2006). Tendo como principais componentes, a sarcopenia, alterações imunológicas e alterações neuroendócrinas (Fried et al., 2001).

O idoso considerado frágil está mais vulnerável a efeitos adversos à sua saúde, tais como: agravamento de doenças, comorbidades, institucionalização, quedas, hospitalização, incapacidade e morte (Fried et al., 2001).

Diante das consequências desencadeadas por esta síndrome, torna-se importante identificar precocemente o idoso frágil ou com predisposição a fragilidade para reabilitá-lo e tentar reverter esta condição. Sendo assim, é de fundamental relevância utilizar instrumentos de fácil aplicação, rápido e simples, capaz de triar o idoso frágil precocemente. Segundo Cooper et al. (2011), diversos indicadores são utilizados na avaliação das condições de saúde da população idosa, dentre eles o desempenho motor ocupa lugar peculiar, pois expressa as habilidades físicas e mentais necessárias à vida independente e autônoma.

Os testes de desempenho motor são medidas objetivas importantes para avaliação da funcionalidade dos idosos, possibilitando a identificação da capacidade física e motora envolvidas nas diversas tarefas relacionadas às atividades de vida diária (Guralnick \& Ferruci, 2003; Seidel, Brayne, \& Jagger, 2011), podendo também auxiliar na execução de programas de intervenção (Barbosa, Miranda, Guimarães, Corseuil, \& Corseuil, 2011).

Diante do exposto, o objetivo deste estudo foi identificar os indicadores de desempenho motor mais fortemente associados à fragilidade e seus respectivos pontos de corte, e verificar o melhor indicador como instrumento de triagem para discriminar fragilidade em idosos cadastrados em Unidade de Saúde da Família.

\section{MÉTODO}

O presente estudo caracteriza-se como observacional de corte transversal, baseado em informações extraídas de um banco de dados de uma pesquisa intitulada "PET- Saúde Jequié: Identificando $\mathrm{o}$ processo saúde-doença em população adstrita às Unidades de Saúde da Família" o qual foi aprovado pelo comitê de ética e pesquisa, parecer $\mathrm{n}^{\circ}$ 084/2011 (CAAE 0064.0.454.000-11). Os dados foram coletados com os idosos cadastrados na Unidade de Saúde da Família (USF) Giserlando Biondi, localizada no bairro Pau Ferro, na cidade de Jequié-Ba, tendo como cenário o domicílio dos idosos.

\section{Participantes}

Foram entrevistados 139 idosos em domicilio, sendo 105 mulheres e 34 homens, com idades $\geq$ 60 anos, capazes de responder ao questionário e realizar os testes de força de preensão manual, teste de equilíbrio, mobilidade e de caminhada. Os idosos que não se adequaram a esses critérios, além dos acamados, amputados, cadeirantes, com Acidente Vascular Cerebral (AVC), com próteses, com dificuldade de entendimento e realização dos testes foram excluídos do estudo.

Antes da coleta de dados, os prontuários dos idosos cadastrados na unidade de saúde foram analisados e aqueles com suspeita de déficit cognitivo, avaliado pelo Mini Exame do Estado Mental (MEEM), foram excluídos. Durante a coleta os idosos foram questionados se nos últimos meses eles se esqueciam das coisas que faziam. Caso o idoso relatasse andar meio esquecido, um membro da família ou cuidador ajudava nas respostas durante a coleta.

\section{Instrumentos}

Os dados foram coletados no período de maio a novembro de 2013, por estudantes de diferentes áreas da saúde, após treinamento prévio, com supervisão de seis preceptores e um tutor do Programa de Educação pelo Trabalho 
para Saúde/PET-Saúde Grupo Idoso I, da Universidade Estadual do Sudoeste da Bahia, por meio de um questionário estruturado, no qual foram questionados os antecedentes pessoais, familiares, doenças associadas, informações sóciodemográficas e tempo gasto realizando atividades físicas usando o Questionário Internacional de Atividade Física- IPAQ, além da realização de medidas antropométricas (massa corporal e estatura) e de alguns testes físicos: Força de preensão manual, teste de sentar e levantar da cadeira, teste de caminhada de 2,44 m e teste de mobilidade. Durante a realização dos testes de desempenho motor, o avaliador explicava e demonstrava para os idosos como os testes seriam realizados.

\section{Procedimentos}

Inicialmente foram mensuradas a massa corporal e estatura. Para a medida da massa corporal, os idosos ficaram de pé, de frente para a escala de medida, sem calçado e com o mínimo de roupa possível. A balança utilizada para a medida da massa corporal foi uma balança digital de plataforma em vidro, da marca G.Tech, com variação de $0,1 \mathrm{~kg}$, capacidade mínima de $100 \mathrm{~g}$ e capacidade máxima de $150 \mathrm{~kg}$.

A estatura corporal foi medida entre a distância do vértex e a região plantar usando um esquadro e uma fita antropométrica inextensível com 2 metros (Sanny). Os idosos foram orientados a permanecerem em posição ortostática, descalços, com os pés unidos, calcanhares em contato com parede e respeitando as orientações do plano de Frankfurt, também foi solicitado aos mesmos que fizessem uma inspiração no momento da mensuração. Com os valores da massa e estatura foi calculado o índice de massa corporal (IMC).

\section{Fragilidade (variável dependente)}

A síndrome da fragilidade foi diagnosticada de acordo com os cinco critérios proposto por Fried et al. (2001), sendo eles: Perda de peso, baixa força muscular, fadiga, lentidão no teste de caminhada e baixo nível de atividade física.

Perda de peso: foi definida por meio do autorrelato da perda de peso não intencional ( $\geq$ $4,5 \mathrm{~kg}$ ou $\geq 5 \%$ do peso corporal no ano anterior).
Baixa força muscular. Foi definida pelo teste de força de preensão manual (FPM) usando um dinamômetro (modelo EH101, e.clear). Para realização do teste o idoso permaneceu sentado, coluna ereta, com os pés apoiados no chão e cotovelo fletido a $90^{\circ}$. Os idosos foram orientados e incentivados a pressionar a alça do dinamômetro exercendo o máximo de força (Figueredo, Sampaio, Mancini, Silva, \& Souza, 2006). Foram realizadas três tentativas com intervalo de 40 segundos, sendo usado para análise o maior valor.

A baixa força muscular foi definida de acordo com o sexo e o índice de massa corporal [IMC = massa corporal $(\mathrm{kg}) /$ estatura $\left.^{2}(\mathrm{~m})\right]$. O IMC foi classificado em três categorias (American Academy of Family Physicians, 2002): $<22$ $\mathrm{kg} / \mathrm{m}^{2}$, baixo peso; $22.0 \leq \mathrm{IMC} \leq 27 \mathrm{~kg} / \mathrm{m}^{2}$, adequado; $>27 \mathrm{~kg} / \mathrm{m}^{2}$, sobrepeso. Para cada categoria, os pontos de corte para a FPM $(\mathrm{kg})$ foi fixado no percentil 25, para homens e mulheres: baixo peso, 21.2 e 10.7 kgf; peso adequado, 24,8 e $14.6 \mathrm{kgf} ; \quad$ sobrepeso 21 e $15.5 \mathrm{kgf}$, respetivamente. Foi considerado critério de fragilidade os idosos que apresentaram baixa força muscular corrigida pelo IMC e aqueles que foram incapazes de realizar o teste devido a limitações físicas.

Fadiga: Foi definida de acordo com a exaustão avaliada por autorrelato de fadiga, indicado por duas questões da Center for Epidemiological Studies - Depression (CES-D) (Batistoni, Neri, \& Cupertino, 2007) : "Você sentiu que teve que fazer esforço para dar conta das suas tarefas habituais?" e "Você não conseguiu levar adiante as suas coisas?". Se o idoso respondeu na "maioria das vezes" e/ou "sempre" para uma das duas questões foi considerado como critério de fragilidade.

Lentidão no teste de caminhada: Foi definida por meio do desempenho físico no teste de caminhada de $2.44 \mathrm{~m}$. Para este teste o idoso foi orientado a caminhar de uma extremidade a outra em sua velocidade habitual, como se estivesse andando pela rua. Os idosos poderiam usar dispositivos de apoio, se necessário, e realizou-se o trajeto duas vezes, com o tempo sendo registrado em segundos, com o menor tempo usado nas análises. $\mathrm{O}$ idoso foi 
considerado capaz de realizar o teste, quando conseguia concluí-lo em um tempo $\leq 60$ (Guralnik et al., 1994).

O tempo gasto para realizar o teste de caminhada foi ajustado de acordo com o sexo e a estatura dos idosos. A estatura foi dividida em duas categorias, com base na mediana (percentil 50): homens $\leq 1.59 \mathrm{~m}$ e mulheres $\leq 1.48 \mathrm{~m}$, abaixo ou igual a mediana; homens $>1.59 \mathrm{~m} \mathrm{e}$ mulheres $>1.48 \mathrm{~m}$, acima da mediana. Para cada categoria, os pontos de corte para o teste de caminhada foi fixado no percentil 75: abaixo ou igual a mediana $\geq 5.3 \mathrm{~s} \mathrm{e} \geq 6$ s; e acima da mediana $\geq 5 \mathrm{~s}$ e $\geq 6.2 \mathrm{~s}$ (para homens e mulheres, respetivamente). Foi considerado como critério de fragilidade os idosos que pontuaram acima do ponto corte no teste de caminhada e aqueles que foram incapazes de realizar o teste devido a limitações físicas.

Baixo nível de atividade física: $\mathrm{O}$ instrumento utilizado para avaliar o nível de atividade física habitual foi o International Physical Activity Questionnaire (IPAQ), versão curta (Craig et al., 2003). Os indivíduos que realizaram menos de 150 minutos por semana em atividades físicas moderadas e/ou vigorosas foram considerados insuficientemente ativos, pontuando como um critério de fragilidade.

Segundo Fried et al. (2001), são considerados "Não-frágeis" os idosos que não apresentaram nenhum dos critérios, "Pré-frágeis" um ou dois dos critérios e "Frágeis" três ou mais dos cinco critérios supracitados. No entanto, para esse estudo a variável dependente, fragilidade, foi dicotomizada em "Não-frágil" para os idosos que apresentaram no máximo dois critérios e "Frágil" para os idosos que apresentaram três ou mais critérios.

Testes de desempenho motor (variáveis independentes)

Força de preensão manual: foi avaliada no braço dominante usando um dinamômetro hidráulico (modelo EH101, e.clear), para testar a força do membro superior. Cujos procedimentos foram descritos anteriormente.

Teste de sentar e levantar da cadeira: foi utilizado para avaliar a força/resistência de membros inferiores. $\mathrm{O}$ idoso foi orientado sobre o teste, e em seguida foi perguntado se o mesmo sentia-se confiante para levantar e sentar rapidamente da cadeira por cinco vezes consecutivas com os braços cruzados sobre o peito. Para a realização do teste foi utilizado uma cadeira de aproximadamente $45 \mathrm{~cm}$, sendo registrado o tempo que o idoso gastou para levantar e sentar da cadeira cinco vezes o mais rápido possível. O idoso foi considerado capaz de realizar o teste, quando conseguia concluí-lo em um tempo $\leq 60$ s (Barbosa et al., 2005).

Teste de caminhada: Foi definida por meio do desempenho físico no teste de caminhada de 2,44 $\mathrm{m}$, cujos procedimentos foram descritos anteriormente.

Teste de mobilidade: A mobilidade/flexibilidade foi avaliada por meio do teste de pegar um lápis. Para esta tarefa, os idosos foram convidados a permanecerem em pé, com os pés lado a lado e, ao comando do entrevistador os mesmos deveriam se abaixar e pegar um lápis, colocado a 30 centímetros à frente dos seus pés, o mais rápido possível e retornar a posição inicial. O idoso foi considerado capaz de realizar o teste, quando conseguia concluí-lo sem qualquer apoio em um tempo $\leq 30$ segundos (Rúben \& Siu, 1990).

\section{Variáveis de ajuste}

As variáveis de ajuste foram: grupo etário (60 - 69, 70 - 79 e $\geq 80$ anos), sexo (masculino e feminino), arranjo familiar (acompanhado e sozinho) e quedas (sim e não). Essas variáveis foram selecionadas e categorizadas de acordo com um estudo prévio, que encontrou associação destas com a fragilidade na amostra investigada (Santos, Fernandes, Cassoti, Coqueiro, \& Carneiro, 2014).

\section{Análise estatística}

Inicialmente realizou-se uma análise descritiva das variáveis (frequências, médias e desvios padrão). A associação entre os testes de desempenho motor e a fragilidade foi testada por meio da técnica de regressão logística binária. Apenas a variável FPM foi estratificada por sexo, devido aos valores entre homens e mulheres serem discrepantes. Foram calculados modelos ajustados para estimar as odds ratio (OR), com 
os seus respectivos intervalos de confiança de 95\% (IC95\%). O poder de diagnóstico da fragilidade dos indicadores de desempenho motor e a identificação dos melhores pontos de corte foram avaliados por meio dos parâmetros fornecidos pela curva Receiver Operating Characteristic (ROC): área sob a curva ROC (ASC), sensibilidade e especificidade.

Em todas as análises o nível de significância adotado foi de $5 \%(\alpha=0,05)$. Os dados foram analisados no IBM SPSS Statistics for Windows (IBM SPSS. 21.0, 2012, Armonk, NY: IBM Corp.) e MedCalc (versão 9.1.0.1, 2006).

\section{RESULTADOS}

Participaram do estudo 139 idosos, sendo 105 $(75,5 \%)$ mulheres e $34(24,5 \%)$ homens. A idade dos idosos variou de 60 a 101 anos, com idade média de 72,32 $\pm 8,4$ anos. A idade média das mulheres foi $72,16 \pm 8,51$ (60 - 101 anos) e dos homens foi $72.85 \pm 8,43$ (60-92 anos). Em relação ao grau de instrução do chefe da família, $73,1 \%$ declararam serem analfabetos ou com ensino médio incompleto, enquanto ao nível sócioeconômico $42 \%$ dos idosos pertence a categoria C e $57,2 \%$ encontrou-se na categoria D e E. A Tabela 1 apresenta as variáveis qualitativas do estudo.

Tabela 1

Análise descritiva das variáveis qualitativas do estudo.

\begin{tabular}{lccc}
\hline Variáveis & \% resposta & $\mathrm{N}$ & $\%$ \\
\hline Grupo etário & 99.3 & & \\
$\quad$ 60-69 anos & & 56 & 40.6 \\
$\quad 70-79$ anos & & 56 & 40.6 \\
$\quad \geq 80$ anos & & 26 & 18.8 \\
Arranjo familiar & 98.6 & & \\
$\quad$ Vive sozinho & & 79 & 56.8 \\
$\quad$ Vive acompanhado & & 60 & 43.2 \\
Quedas & 99.3 & & \\
$\quad$ Sim & & 38 & 27.5 \\
$\quad$ Não & & 100 & 72.5 \\
Fragilidade & 97.8 & & \\
$\quad$ Frágil & & 23 & 16.9 \\
$\quad$ Não frágil & & 113 & 83.1 \\
\hline
\end{tabular}

A Tabela 2 apresenta as variáveis quantitativas do estudo. Apenas a variável força muscular foi estratificada pelo sexo devido aos valores discrepantes entre homens e mulheres.

A Tabela 3 mostra a OR para fragilidade a partir dos testes de desempenho motor. O modelo de regressão logística (ajustado pelo grupo etário, sexo, arranjo familiar e quedas) apontou que apenas o tempo gasto para a realização do teste de caminhada foi positivamente associado à fragilidade $(\mathrm{p}<0,01)$, indicando que cada incremento de $1 \mathrm{~s}$ no tempo de realização deste teste aumenta $30 \%$ a probabilidade de fragilidade em idosos.

O teste de força de preensão manual no sexo feminino foi inversamente associado à fragilidade $(\mathrm{p}<0,001)$, indicando que o aumento em uma unidade da FPM (1kgf) diminui em aproximadamente $26 \%$ a probabilidade de fragilidade em mulheres idosas.

Tabela 2

Análise descritiva das variáveis quantitativas do estudo.

\begin{tabular}{lcccc}
\hline Variáveis & $\begin{array}{c}\% \\
\text { resposta }\end{array}$ & Média & DP & Mín-máx \\
\hline FPM - M (kgf) & 100 & 27.1 & 6.9 & $9.9-40.6$ \\
FPM - F (kgf) & 97.1 & 17.1 & 5.4 & $5.4-29.7$ \\
Sentar e levantar (s) & 83.5 & 16.3 & 6.3 & $6.1-50.4$ \\
Caminhada (s) & 90.6 & 5.40 & 2.9 & $2-18$ \\
Pegar um lápis (s) & 79.9 & 3.94 & 2.6 & $1-14$ \\
\hline
\end{tabular}

FPM: Força de preensão manual; M: masculino; F: feminino; DP: desvio padrão

Tabela 3

Odds ratio para testes de desempenho motor $e$ fragilidade em idosos.

\begin{tabular}{llcc}
\hline Variáveis & OR $^{*}$ & IC95\% & p-valor \\
\hline FPM - M & 0.84 & $0.69-1.02$ & 0.08 \\
FPM - F & 0.74 & $0.63-0.86$ & $<0.001$ \\
Sentar e levantar & 1.02 & $0.94-1.10$ & 0.53 \\
Caminhada & 1.30 & $1.09-1.53$ & $<0.01$ \\
Pegar um lápis & 1.12 & $0.93-1.36$ & 0.21 \\
\hline
\end{tabular}

OR: odds ratio; FPM: Força de preensão manual; M: masculino; F: feminino

* Ajustado para grupo etário, sexo, arranjo familiar e quedas.

Tabela 4

Pontos de corte, sensibilidade e especificidade dos indicadores de desempenho motor como discriminadores de fragilidade em idosos.

\begin{tabular}{lccc}
\hline \multicolumn{1}{c}{ Variáveis } & $\begin{array}{c}\text { Ponto de } \\
\text { corte }\end{array}$ & Sensibilidade & Especificidade \\
\hline FPM - F (kgf) & 14.6 & 83.3 & 79.0 \\
T. Caminhada (s) & 5 & 88.9 & 74.5 \\
\hline
\end{tabular}

FPM-F; força de preensão manual sexo feminino; kgf: quilos força; T: teste; s: segundos.

A Tabela 4 mostra os valores de sensibilidade do teste de caminhada $(88,9 \%)$ e da FPM-F $(83,3 \%)$, demonstrando adequada capacidade de identificar os idosos portadores com a síndrome de fragilidade que ultrapassaram os valores dos pontos de corte (verdadeiros positivos). Os valores de especificidade do teste de caminhada 
foi $74,5 \%$ e da FPM-F 79,0\%, demonstrando adequada capacidade de identificar os idosos não portadores da síndrome de fragilidade que não ultrapassaram os valores dos pontos de corte (verdadeiros negativos).

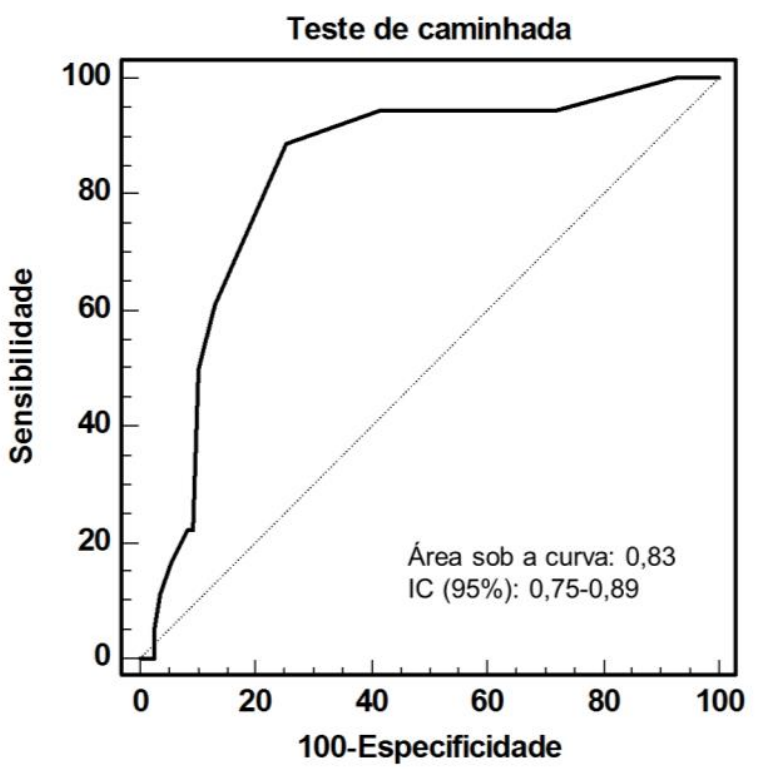

a
Na Figura 1 encontram-se as áreas sob a curva ROC do teste de caminhada para ambos os sexos (figura 1a) e da FPM para as mulheres (figura 1b). Observou-se um valor satisfatório das áreas sob a curva ROC (>70\%) para ambos os testes.

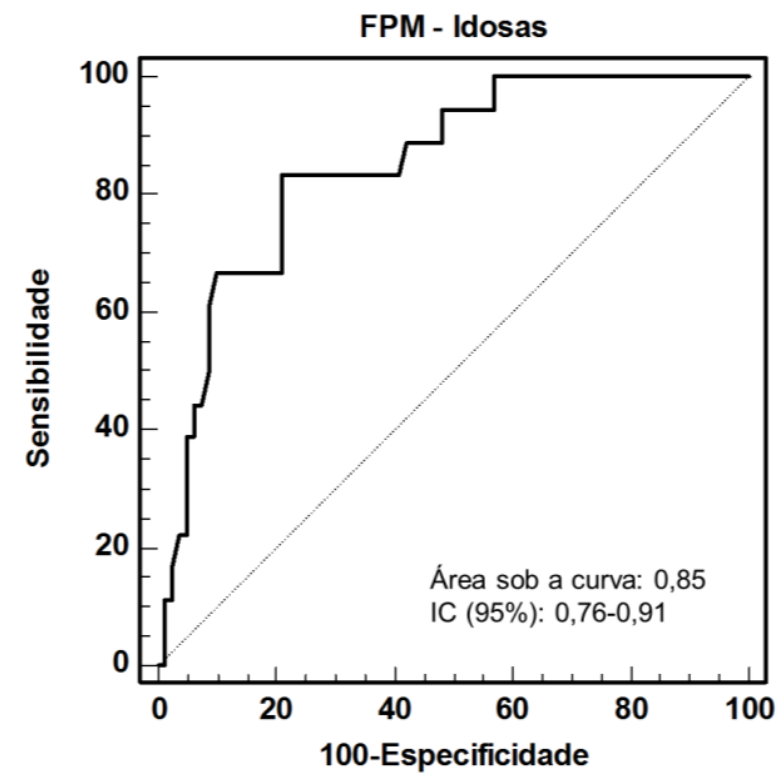

$b$

Figura 1. Curvas ROC (a): comparando o teste de caminhada como discriminador de fragilidade em idosos; (b) comparando a FPM como discriminadora de fragilidade apenas no grupo das mulheres idosas.

\section{DISCUSSÃO}

Este estudo se propôs a identificar se os indicadores de desempenho motor poderiam ser utilizados como preditores de fragilidade em idosos. Até o momento, esse foi primeiro estudo nacional que usou testes de desempenho motor como indicadores de triagem para identificar idosos frágeis cadastrados em Unidade de Saúde da Família.

No presente estudo, a prevalência de idosos frágeis foi de 16,9\%. Em outros estudos nacionais a prevalência de fragilidade variou entre $20 \%$ e $23,8 \%$ (Silva, Vieira, Arantes, \& Dias, 2009; Junior et al.,2014). As possíveis explicações para a variação na prevalência de fragilidade em idosos, são: os diferentes instrumentos usados para avaliar os critérios de fragilidade e as disparidades nas composições das amostras relacionadas à etnia e questões socioeconômicas (Tribress \& Oliveira, 2011).

Os principais achados deste estudo foram às associações entre a fragilidade e o tempo gasto no teste de caminhada em ambos os sexos, e com a força de preensão manual no sexo feminino (FPM-F). O presente estudo também encontrou uma alta sensibilidade e especificidade em ambos os testes, indicando que os idosos que realizarem o teste de caminhada de $2,44 \mathrm{~m}$ em um tempo $\geq$ 5 segundos e a FPM-F $\leq 14,6$ (kgf) serão fortes candidatos à condição de fragilidade.

Em um estudo realizado por Pinedo et al. (2009) com 246 idosos, os autores observaram uma associação positiva entre menor velocidade de marcha e fragilidade em mulheres, corroborando com atual estudo. Diversos autores apontam que a avaliação da velocidade de marcha tem sido o preditor de fragilidade com maior confiabilidade, praticidade e de baixo custo, sendo capaz de identificar o idoso com fragilidade (Cesari et al.,2005; Pinedo, Saavedra, \& Jimeno, 2009; Rothman, Leo-Summers, \& Gill, 2008; Van Kan et al., 2010).

No entanto, são escassos na literatura estudos que apontem pontos de cortes do tempo no teste de caminhada 2,44 $\mathrm{m}$ com intuito de triar idosos frágeis. Sendo assim, este teste de caminhada 
torna-se uma ferramenta importante, por ser um teste simples, barato e de fácil aplicação e que pode ser aplicado como uma forma de triagem na prática clínica ou até mesmo na própria residência do idoso.

Alguns estudos como o de Pinedo, Saavedra, e Jimeno (2009), encontraram um ponto de corte de $0,7 \mathrm{~m} / \mathrm{s}$, enquanto o estudo de Cesari et al. (2005) definem como limite $1 \mathrm{~m} / \mathrm{s}$. Dessa forma, observa-se disparidades nos resultados, não existindo valores plenamente estabelecidos para velocidade da marcha ideal na identificação da presença de fragilidade. Os resultados dos estudos supracitados não podem ser comparados aos achados do presente estudo, porque os parâmetros avaliados são diferentes.

Mesmo com indefinições de ponto de corte, autores acreditam que a velocidade de marcha fornece uma avaliação mais adequada, sendo este o único item dos critérios de fragilidade a ser implementado na prática clínica, funcionando como um instrumento altamente confiável, capaz de identificar idosos com riscos clínicos por mais tempo (Van Kan et al., 2010).

A força de preensão manual tem sido considerada uma ferramenta importante para avaliação da fragilidade, sendo este indicador, considerado um significante preditor de autopercepção, fadiga, incapacidade, morbidade, e mortalidade (Van Kan et al., 2010).

Os valores de FPM definidos por estudos prévios demonstraram que valores iguais ou inferiores a $20 \mathrm{~kg}$ relacionam-se, de forma independente, com risco para dependência futura e baixos níveis de saúde (Jylha, Guralnik, Balfour, \& Fied, 2001).

Esse teste vem sendo utilizado como indicador de força global e de funcionalidade, a diminuição deste indicador, explica que os idosos são sedentários, apresentam déficit de massa corporal, problemas de saúde e limitações funcionais em atividades que exijam participação dos membros superiores e inferiores (Kuh, Bassey, Butterworth, Hardy, \& wadsworth, 2005).

Com características semelhantes em relação a predomínio do gênero feminino, o estudo de Bez e Neri (2014), observou que as mulheres tinham menos força de preensão manual, onde tiveram a maior probabilidade de serem consideradas como portadoras de baixa força de preensão. Relacionando esse resultado do estudo de Bez e Neri (2014) com os resultados do atual estudo, explica que essa baixa força de preensão manual ocasionaria a probabilidade de essas idosas serem frágeis. Uma limitação do estudo está relacionada ao seu delineamento transversal, o qual não foi possível constituir uma relação causal entre a síndrome da fragilidade e o desempenho motor em idosos. Por outro lado, destaca-se como ponto forte do estudo o uso de um indicador de desempenho motor rápido, prático de baixo custo para triar precocemente idosos com características de fragilidade cadastrados em unidades de saúde.

\section{CONCLUSÃO}

Com base nos resultados, conclui-se que houve uma associação positiva entre a fragilidade e o tempo gasto no teste de caminhada nos idosos de ambos os sexos, e uma associação negativa entre a fragilidade e a força de preensão manual nos idosos do sexo feminino. O teste de caminhada apresentou-se como o melhor indicador de triagem para discriminar a fragilidade em idosos cadastrados em uma Unidade de Saúde da Família.

\section{Agradecimentos:}

Ao Programa de Educação pelo Trabalho para Saúde (PET-Saúde), Grupo Idoso I, pelo apoio e colaboração.

Conflito de Interesses:

Nada a declarar.

\section{Financiamento:}

Nada a declarar

\section{REFERÊNCIAS}

Alvarado, B. E., Zunzunegui, M. V., Béland, F., \& Bamvita, J. M. (2008). Life course social and health conditions linked to frailty in Latin American older men and women. The journals of gerontology Series A, Biological sciences and medical sciences, 63, 1399-1406.

American Academy of Family Physicians, American Dietetic Association, National Council on the Aging. Nutrition screening e intervention resources for healthcare professionals working 
with older adults. Nutrition Screening Initiative. Washington: American Dietetic Association; 2002. Disponível em: <http://www.eatright.org/cps/rde/xchg/ada/hs .xsl/nutrition_nsi_enu_html.htm $>$.

Barbosa, A. R., Miranda, L. M., Guimarães, A. V., Corseuil, H. X., \& Corseuil, M. W. (2011) Age andgender differences regarding physical performance in the elderly from Barbados and Cuba. Revista de Saúde Pública, 13(1), 54-66.

Barbosa, A. R.; Souza JMP; Lebrão ML; Laurenti R; Maria de Fátima N. Marucci MFN. Functional limitations of Brazilian elderly by age and gender differences: data from SABE Survey. Cad. saúde pública; 2005, 21(4): 1177-85.

Batistoni, S. S. T., Neri, A. L., \& Cupertino, A. P. F. B. (2007). Validade da escala de depressão do Center for Epidemiological Studies entre idosos brasileiros. Revista de Saúde Pública, 41, 598605. doi.org/10.1590/S003489102007000400014

Bez, J. P. O., \& Neri, A. L. (2014). Velocidade da Marcha, força de preensão e saúde percebida em idosos: dados da rede FIBRA de campinas. Ciência \& Saúde Coletiva, 19(8), 3343-3353. doi: 10.1590/1413-81232014198.09592013

Cesari, M., Kritchevsky, S. B., Baumgartner, R. N., Atkinson, H. H., Penninx, B. W., Lenchik, L.,... Pahor, M. (2005). Sarcopenia, obesity, and inflammation-results from the trial of angiotensin converting enzyme inhibition and novel cardiovascular risk factors study. The American Journal of Clinical Nutrition, 82 (2), 428-34.

Cooper, R., Kuh, D., Cooper, C., Gale, C. R., Lawlor, D. A., Matthews, F., Hardy, R.,...FALCon and HALCyon Study Teams. (2011). Objective measures of physical capability and subsequent health: a systematic review. Journal Age and Ageing, $\quad 40(1), \quad 14-23$. doi: 10.1093/ageing/afq117

Craig, C. L., Marshall, A. L., Sjöström, M., Bauman, A. E., Booth, M. L., Ainsworth, B. E.,..Oja, P. (2003). International Physical Activity Questionnaire: 12-country reliability and validity. Medicine \& Science in Sports \& Exercise, 35, 1381-1395.

Figueiredo, I. M., Sampaio, R. F., Mancini, M. C.,Silva, F. C. M.,Souza, M. A. P., \& Peixoto, M. A.(2006).Teste de forca de preensão utilizando o dinamômetro Jamar. Revista Acta Fisiátrica, 14(2), 104-110.

Fried, L. P., Tangeon, C. M., Walston, J., Newman, A. B., Hirsch, C., Gottdiener, J.,... McBurnie, M. A. (2001). Frailty in older adults: evidence for a phenotype. Journals of gerontology Series $A$, Biological sciences and medical sciences, 56, 146156.

Fried, L. P., Ferrucci, L., Darer, J., Williamson, J. D., \& Anderson, G. (2004). Untangling the concepts of disability, frailty, and comorbidity: implications for improved targeting and care. The Journals of gerontology Series A, Biological sciences and medical sciences, 59, 255-263.

Guralnik, J. M., Simonsick, E. M., Ferrucci, L., Glynn, R. J., Berkman, L. F., Blazer, D. G., Scherr, P.A., Wallace, R.B. (1994). A short physical performance battery assessing lower extremity function: association with self-reported disability and prediction of mortality and nursing home admission. Journal of Gerontology, 49(2), 85-94.

Junior, W. M. R., Carneiro, J. A. O., Coqueiro, R. S., Santos, K. T., \& Fernandes, M. H. (2014). Préfragilidade e fragilidade de idosos residentes em município com baixo Índice de Desenvolvimento Humano. Revista Latino-Americana de Enfermagem, 22(4), 654-661.

Jylha, M., Guralnik, J. M., Balfour, J., Fried, L. P. (2001). Walking difficulty, walking speed, and age as predictors of self-rated health: The Women's Health and Aging Study. The Journals of gerontology Series A, Biological sciences and medical sciences, 56, 609-617.

Kuh, D., Bassey, E. J., Butterworth, S., Hardy, R., \& Wadsworth, M. E.(2005). The Musculoskeletal Study Team. Grip strength, postural control, and functional leg power in a representative cohort of British men and women: associations with physical activity, health status, and socioeconomic conditions. The Journals of gerontology Series A, Biological sciences and medical sciences, 60, 224-31

Pinedo, L. F. V., Saavedra, P. J. O., \& Jimeno, H. A. C. (2009). Velocidad de la marcha em adultos mayores de La comunidad en Lima, Perú. Revista Medica Herediana, 20(3), 133-138

Rockwood, K., Howlett, S. E., MacKnight, C., Beattie, B. L., Bergman, H., Hébert, R., McDowell, I. (2004). Prevalence, attributes, and outcomes of fitness and frailty in community-dwelling older adults: report from the Canadian study of health and aging. The journals of gerontology Series $A$, Biological sciences and medical sciences, 59(12), 1310-7.

Rothman, M. D., Leo-Summers, L., \& Gill, T. M.(2008). Prognostic Significance of potencial Frailty Criteria. Journal of the American Geriatrics Society, 56(12), 2211-116. doi: 10.1111/j.1532-5415.2008.02008.x.

Rúben, D.B., Siu, A.L. (1990). Uma medida objetiva da função física dos idosos ambulatoriais. Journal of the American Geriatrics Society, 38(10), 11051112.

Santos, P.H.S., Fernandes, M.H., Cassoti, C.A., Coqueiro, R.S., Carneiro, J. A. O. (2014). The profile of fragility and associated factors among the elderly registered in a Family Health Unit. Ciências e Saúde Coletivas, 20(6), 1917-1924. doi: 10.1590/1413-81232015206.17232014

Santos-Eggimann, B., Cuénoud, P., Spagnoli, J., \& Junod, J. (2009). Prevalence of frailty in middleaged and older community-dwelling Europeans living in 10 countries. The journals of gerontology Series A, Biological sciences and 
96 | PLS Santos, MH Fernandes, PHS Santos, TDB Santana, CA Cassoti, RS Coqueiro, JAO Carneiro

medical sciences, 64, 675-681. doi: 10.1093/gerona/glp012

Seidel, D; Brayne, C., \& Jagger, C. (2011). Limitations in physical functioning among olderpeople as a predictor of subsequent disability in instrumental activities of daily Living. Journal Age and Ageing, 4O(4), 463-9. doi: 10.1093/ageing/afr054

Silva, S. L. A., Vieira, R. A., Arantes, P., \& Dias, R. C. (2009). Avaliação de fragilidade, funcionalidade e medo de cair em idosos atendidos em um serviço ambulatorial de Geriatria e Gerontologia.Fisioterapia e Pesquisa, 16(2), 120125.

Sousa, A. C., Dias, R. C., Maciel, Á. C., \& Guerra, R. O. (2012). Frailty syndrome and associated factors in community-dwelling elderly in Northeast Brazil. Archives of Gerontology and Geriatrics, 54, 95-101. doi: 10.1016/j.archger.2011.08.010
Tribess, S., \& Oliveira, R. J. (2011). Síndrome da fragilidade biológica em idosos: revisão sistemática. Revista de Saúde Pública, 13(5), 853-64. doi.org/10.1590/S012400642011000500014

Van Kan, G. A., Rolland, Y., Houles, M.,GilletteGuyonnet, S., Soto, M., \& Vellas, B. (2010). The Assessment of Frailty in olderAdults. Clinics in Geriatric Medicine, 26(2), 275-286. doi:10.1016/j.cger.2010.02.002.

Vieira, R. A., Guerra, R. O., Giacomin, K. C., Vasconcelos, K. S. S., Andrade, A. C. S., Pereira, J. M. D.,... Dias, R. C. (2013). Prevalência de fragilidade e fatores associados em idosos comunitários de Belo Horizonte, Minas Gerais, Brasil: dados do Estudo FIBRA. Caderno de Saúde Pública, 29(8), 1631-1643. doi.org/10.1590/0102-311X00126312.

Walston, J. D. (2006). Frailty as a model of aging. In: Conn PM, editor. Handbook of models for humon aging. Elsevier Academic Press, 697-702.

Todo o conteúdo da revista Motricidade está licenciado sob a Creative Commons, exceto quando especificado em contrário e nos conteúdos retirados de outras fontes bibliográficas. 